\title{
Geriatric renal palliative care is coming of age
}

\author{
Mark Swidler
}

Received: 5 August 2010/Accepted: 6 August 2010/Published online: 20 August 2010

(C) Springer Science+Business Media, B.V. 2010

In 2003, Oreopoulos and Dimkovic called attention to the growing geriatric renal population, the need for a re-assessment of their management and aptly stated that "Geriatric nephrology is coming of age" [1]. Since that prescient observation, the dialysis experience in the elderly population has produced some somber facts. Understanding how to best treat these patients has assumed primary importance since dialysis units will soon be overpopulated by those older than 75 years, and the great majority will not receive transplants. The course for an individual patient remains uncertain even with the best prognostic tools. The future predicts an increasing elderly renal population whose main desire is to maintain independence and quality of life (QOL) through optimal mental capacity and physical functioning despite mounting comorbidities, geriatric syndromes and a shrinking personal world. How can we best prepare to meet this challenge? Is there an approach that engenders hope? A shift in perspective provides a starting point.

M. Swidler $(\square)$

Mount Sinai School of Medicine, 1 Gustave

L. Levy Place, New York, NY 10029, USA

e-mail: mark.swidler@mssm.edu

\section{The landscape of geriatric CKD and dialysis is a model of chronic progressive illness}

The 1-year probability of death and hospitalization in the general dialysis population is 24 and $66 \%$, respectively [2]. This compares with a first-year mortality of $46 \%$ in those over the age of 80 that does not measurably change over time [3]. Added risk factors for poor outcomes include increasing age, body mass index $(\mathrm{BMI})<20$, low serum albumin, inability to transfer or ambulate, $\geq 4$ comorbid conditions, peripheral vascular disease and congestive heart failure $[2,3]$. Geriatric syndromes like frailty, functional disability, cognitive dysfunction, depression and falls progress with advancing CKD and ESRD. They too are powerful predictors of adverse consequences like mortality, hospitalization, nursing home placement and hip fractures (REF) The combination of geriatric syndromes and dialysis will have synergistic effects.

Dialysis is a life-sustaining therapy with the attributes of a chronic progressive illness. This includes a reduced lifespan for those starting dialysis when compared with age-matched controls in the general population, progressive functional decline over months to years, episodes of acute complications with repeated hospitalizations, significant comorbidity, high symptom burden and caregiver stress. This is the "ESRD patient disease trajectory" that shares similarities with advanced heart failure and lung disease [4]. Renal replacement may correct uremia but the trajectory continues. 
Since the course is uncertain for any individual dialysis patient, the care plan must be dynamic, individualized and appropriately updated.

The nursing home ESRD dialysis patient has three times the annual mortality rate when compared with its non-nursing home dialysis counterpart [5]. Sadly, dialysis in these patients does not appear to stop the loss of functional ability, which is as high as $83 \%$ at the end of 1 year with an associated mortality of $58 \%$ [6]. Of equal concern, impaired decisional capacity is found in up to $60 \%$ of nursing home patients, and among them only $35 \%$ have advanced care directives and 30\% "do not resuscitate" (DNR) orders [5]. This makes informed shared decision-making, addressing goals of care and medical treatment both challenging and time-consuming in this population.

\section{The relief of suffering must be a priority of geriatric nephrology}

The chronic progressive illness model is associated with increasing levels of patient suffering and family distress. To address suffering, it must first be recognized. Suffering has been defined as "a specific state of distress that occurs when the intactness or integrity of the person is threatened or disrupted and involves a symptom (physical or other) or process that threatens the patient's integrity because of fear, the meaning of the symptom, and concerns about the future"[7]. Since it is framed and defined in the particular patient's terms, the same symptom (like chronic pain) or process (like chronic dialysis) may produce suffering in one patient but not in another. This means that nephrology must move beyond traditional renal-specific goals, such as dialysis adequacy and PTH control, appreciate potential causes of suffering, and provide individualized assessment and treatment of these sources.

This includes addressing the multitude of symptoms (such as pain, fatigue, insomnia, depression, poor physical function, anorexia, constipation, pruritus, restless legs) that ESRD patients experience similar to cancer patients $[8,9]$. This symptom burden will be present in varying degrees whether the older patient is in CKD stage 5, recently started on dialysis, on chronic dialysis, or on contemplating dialysis withdrawal. Untreated, it will foster an environment where suffering develops and self-propagates. Similarly, unexplored patient personal goals (e.g., "pain-free"; stay at home; live as long as possible; a "meaningful" life as defined by that individual) without investigation into the patient's personal narrative will miss potential emotional and existential sources of suffering. How can the nephrology community deliver renal care while addressing what is most important to their geriatric patients?

\section{Palliative care addresses sources of suffering in serious progressive illness}

The consensus definition and goal of palliative care put forth by the National Consensus Project for Quality Palliative Care "is to prevent and relieve suffering and to support the best possible quality of life for patients and their families, regardless of the stage of the disease or the need for other therapies" [10]. It is a patient and family-oriented philosophy of care with defined operational guidelines that optimize quality of life by anticipating, preventing and treating suffering. It addresses physical, psychosocial and spiritual needs and focuses on the "person" through a holistic interdisciplinary team approach. It is important to conceptualize the palliative care umbrella as consisting of two separate non-synonymous but fluid subdivisions: non-hospice- and hospice-associated palliative care. Non-hospice palliative care (e.g., treatment of pain and non-pain symptoms; initiation of advance care planning process) is appropriate to introduce at any point in a patient with serious progressive illness and can be provided simultaneously with life-prolonging or restorative treatment (e.g., chronic dialysis or chemotherapy). Understanding this 2 component subdivision is important because palliative care interventions have been wrongly equated with only end-of-life and hospice care thus overshadowing their benefits in patients concurrently receiving life-prolonging therapy. Hospice is a form of palliative care that provides care at the end-of-life (weeks to months). It too is a philosophy of care, underutilized and has unfortunately been narrowly defined through the Medicare Hospice Benefit. Eligibility requires a $2 \mathrm{MD}$-certified prognosis of less than 6 months and a switch from Medicare-covered curative/life-prolonging therapies to the hospice benefit, which can include targeted therapy (e.g., palliative radiotherapy or in some instances palliative ultra filtration) if it is prescribed to treat symptoms not 
associated with the primary diagnosis used for hospice inclusion. The key point is that palliative care is appropriate in all patients with serious illness (high symptom burden, shortened survival, significant comorbidity) at any time along their medical trajectory. This concept has been recently designed as the "simultaneous or shared care model" tailoring medical treatment to patient and family goals $[11,12]$. The focus and relative weight placed on each component of total (curative/restorative and palliative) patient care changes as the equilibrium between disease progression, treatment benefits versus burdens, functional and frailty status, and patient and family goals shifts in a dynamic fashion. Eventually, restorative care is fully replaced by palliative care as the burdens of targeted therapy consistently outweigh the benefits, and patient and their families are faced with the final chapter.

\section{Palliative care provides an integrated treatment plan for the elderly renal patient}

Operationally, palliative care is an agreed upon personal and individualized management plan to optimize quality of life and relieve suffering offered simultaneously with all other appropriate medical therapy. It is suitable in both those undergoing dialysis and nondialysis therapy. Palliative care includes [13-16]:

- Advance care planning-a process of ongoing communication to update prognosis, goals of care and individual life and quality preferences/as the trajectory of decline progresses and end-of-life issues become more prominent

- Symptom control (especially controlling pain with assurance that the use of opioids can be expertly managed)

- Patient and family support to:

- create a sense of control over patient's health care

- relieve potential burdens on loved one

- strengthen interfamily relationships

- Hospice referral when appropriate that allows:

- realization of patient and family wishes

- accelerated holistic management as status changes

- avoidance of unnecessary hospitalizations

- a dignified death in the person's chosen location

\section{Palliative care offers a means to navigate through the dialysis decision process}

Although dialysis is life-sustaining therapy and extends life, it may also create, increase or prolong suffering while not restoring or maintaining wellbeing, function or cognition. It may ultimately prolong the dying process, which is a qualitative state of being that implies progressive unidirectional deterioration and restriction in multiple domains. Recent studies suggest that dialysis may not offer a survival advantage in patients over the age of 75 with multiple comorbidities and cardiac ischemia [17].

The dialysis decision process is a model for patients with any serious chronic disease facing issues with life-sustaining therapy (including mechanical ventilation, pressors, feeding tubes, intravenous hydration, high-risk vascular surgery, aggressive chemotherapy in advanced cancer). A palliative care template creates an environment in which these discussions can be initiated and pursued. It uses a language of hope grounded in reality. The phraseology can set a more empathic tone that allows better understanding of options. It might include phrases such as:

- to forgo (to let pass; to do without) instead of to withhold/draw, which implies taking therapy away or not allowing inclusion

- "non-dialysis therapy" or "kidney non-dialysis medical treatment" instead of "conservative" therapy

- "sub-optimal or non-ideal" instead of "poor" candidate for dialysis

- "refocusing" or "re-orienting" the plan or management objectives" instead of "little or nothing else to do"

- "aggressively" treating symptoms particularly pain with skilled and appropriate use of opioids (no longer called "narcotics")

Traditionally, the choice for CKD patients has been articulated as dialysis versus no dialysis or "conservative" treatment as if "yes" implied "therapy" and the "no" decision implied no or less therapy. This has unknowingly setup dialysis as the standard of care for CKD stage 5 . Dialysis decisions can be better presented as choices between 2 complete therapies (Dialysis or non-dialysis therapy). This signals to patients 
and families that they can receive aggressive care even if they opt to forego dialysis. The discussion of "kidney non-dialysis medical treatment" should emphasize that patients are exercising informed consent and self autonomy; choosing therapy focused on quality of life over quantity of time lived but also simultaneously receiving targeted medical renal therapy.

Non-dialysis medical therapy is also associated with fewer hospitalizations and more deaths at home, which is a concern for elderly frail patients and their families. There is a growing literature that supports this treatment modality as an acceptable choice if nephrologists can work with palliative medicine specialists $[18,19]$. As the trajectory progresses, hope is redefined as making each day as good as possible, achieving an acceptable symptom burden and creating a humane and dignified experience for the patient and family.

The decision to forego life-sustaining therapy has powerful symbolic meaning for family members especially if their loved ones are cognitively impaired. Although it might make sense medically, they may still struggle as the uremic process unfolds. Consistent demonstration of caring, respect and concern by the palliative medicine team will soften this emotional process and increase the potential for acceptable endof-life experience. The medically treated ESRD patient will require increasing home services and transition into a hospice system (home, nursing home with hospice or inpatient hospice depending on goals of care and adequacy of symptom control). Palliative care as part of a simultaneous model approach will help achieve these goals.

\section{Renal geriatric palliative care is coming of age}

Without compromising core beliefs and treatment standards, nephrologists can further expand "best care" to encompass the complex and myriad needs of an aging $\mathrm{CKD} /$ dialysis population and create a treatment plan that will also be medically efficacious. Whether the patient opts for dialysis or non-dialysis therapy is less important than implementing a strategy that offers the best possible outcome based on patient preferences and quality of life. This is the goal of geriatric renal palliative care. The "before" and "after" plan that frames the dialysis decision must remain consistent in a patient-oriented and holistic manner. This means baseline and timely updated geriatric assessment and functional status review. It means using palliative care tools such as symptom evaluation and advance care planning education especially the health care proxy process so patients will be truly represented and have real advocates when they can no longer make their own medical decisions. It means patient and caregiver psychosocial support and regular goals of care review particularly when new acute events and changes in functional status have occurred. In the current dysfunctional health system, the nephrology community faced with an increasing population older adults with multiple geriatric syndromes and comorbidity can substantially improve patient care and outcomes by incorporating the simultaneous care model in standard geriatric renal care.

\section{References}

1. Oreopoulos DG, Dimkovic N (2003) Geriatric nephrology is coming of age. J Am Soc Nephrol 14(4):1099-1101

2. United States Renal Data System (USRDS) 2002

3. Kurella M et al (2007) Octogenarians and nonagenarians starting dialysis in the United States. Ann Intern Med 146(3):177-183

4. Lorenz KA et al (2008) Evidence for improving palliative care at the end of life: a systematic review. Ann Intern Med 148(2):147-159

5. United States Renal Data System (USRDS) 2004

6. Kurella Tamura M et al (2009) Functional status of elderly adults before and after initiation of dialysis. N Engl J Med 361(16):1539-1547

7. Cassell EJ (1999) Diagnosing suffering: a perspective. Ann Intern Med 131(7):531-534

8. Weisbord SD et al (2003) Symptom burden, quality of life, advance care planning and the potential value of palliative care in severely ill haemodialysis patients. Nephrol Dial Transplant 18(7):1345-1352

9. Murtagh FE, Addington-Hall J, Higginson IJ (2007) The prevalence of symptoms in end-stage renal disease: a systematic review. Adv Chronic Kidney Dis 14(1):82-99

10. The National Consensus Project for Quality Palliative Care (2009) Clinical Practice Guidelines for Quality Palliative Care, 2nd Edn

11. Selman LE et al (2009) Palliative care: based on neither diagnosis nor prognosis, but patient and family need. Commentary on Chattoo and Atkin. Soc Sci Med 69(2): 154-157 discussion 158-9

12. Meier DE (2010) The development, status, and future of palliative care. In: Meier DE, Isaacs SL, Hughes RG (eds) Palliative care transforming the care of serious illness. Robert Wood Johnson Foundation Series on Health Policy. Jossey-Bass 
13. Holley JL (2007) Palliative care in end-stage renal disease: illness trajectories, communication, and hospice use. Adv Chronic Kidney Dis 14(4):402-408

14. Brown EA, Chambers EJ, Eggeling C (2008) Palliative care in nephrology. Nephrol Dial Transplant 23(3): 789-791

15. Holley JL (2005) Palliative care in end-stage renal disease: focus on advance care planning, hospice referral, and bereavement. Semin Dial 18(2):154-156

16. Moss AH et al (2004) Palliative care. Am J Kidney Dis 43(1): 172-173
17. Murtagh FE et al (2007) Dialysis or not? A comparative survival study of patients over 75 years with chronic kidney disease stage 5. Nephrol Dial Transplant 22(7): 1955-1962

18. Smith C et al (2003) Choosing not to dialyse: evaluation of planned non-dialytic management in a cohort of patients with end-stage renal failure. Nephron Clin Pract 95(2): c40-c46

19. De Biase V et al (2008) Prolonged conservative treatment for frail elderly patients with end-stage renal disease: the Verona experience. Nephrol Dial Transplant 23(4): 1313-1317 